\title{
Preparation of New $\alpha$-Aminophosphonate Derivatives by Kabachnik-Fields Reaction Using a Recyclable Catalyst
}

\author{
Nellisara D. Shashikumar \\ Department of Chemistry, Sahyadri Science College (Autonomous), Shimoga, Karnataka 577203, India \\ Correspondence should be addressed to Nellisara D. Shashikumar; shashikumarnd@gmail.com
}

Received 21 May 2013; Revised 29 June 2013; Accepted 29 June 2013

Academic Editor: John CG Zhao

Copyright ( 2013 Nellisara D. Shashikumar. This is an open access article distributed under the Creative Commons Attribution License, which permits unrestricted use, distribution, and reproduction in any medium, provided the original work is properly cited.

A convenient and efficient synthetic method for the preparation of some new $\alpha$-aminophosphonate derivatives via a one-pot threecomponent system has been achieved using Amberlite IRC-748 as a recyclable catalyst. This method not only provides an excellent complement for the synthesis of $\alpha$-aminophosphonates but also avoids the use of hazardous acids or expensive/toxic Lewis acids and harsh reaction conditions. Most of the synthesized compounds (4a-o) exhibited activity against bacteria/fungi strains and moderate DPPH radical scavenging activity.

\section{Introduction}

Organophosphorus compounds are ubiquitous in nature and find applications in the fields of agriculture, medicine, and industry [1-3]. Some organophosphorus compounds are important pesticides [4], bactericides [5-7], and antibiotics [5]. Phosphorus analogues of $\alpha$-pyrones act as HIV protease inhibitors [8]. $\alpha$-Aminophosphonic acids constitute important motifs among the organophosphorus compounds in medicinal chemistry due to their obvious structural similarities to $\alpha$-amino acids $[9,10]$. Many natural and synthetic aminophosphonic acids and their ester and peptide derivatives display a wide range of biological activities [11, 12], act as herbicides [13], enzyme inhibitors [14], and antibacterial [15, 16], antiviral [10], and antitumor [17] agents, and may even be peptide mimics [18].

The most common synthetic route to $\alpha$-aminophosphonic acids is via chemical manipulation of the corresponding $\alpha$-aminophosphonates [19-21]. The hydrophosphonylation of imines is a widely used method for the synthesis of $\alpha$-aminophosphonates [19-27]. This is achieved by one of two pathways: (i) in a two-component fashion known as the Pudovik reaction $[28,29]$ or (ii) by the Kabachnik-Fields reaction $[22,23,30,31]$ which combines in situ formation of imine by condensation of amines with an aldehyde or ketone and an hydrophosphonylation step [32].
One-pot Kabachnik-Fields reaction can be promoted by acidic or basic catalysts, microwave irradiation, or by heating [33]. Several Lewis acid catalysts, such as $\mathrm{InCl}_{3}$ [34], $\mathrm{LiClO}_{4}$ $[35,36], \mathrm{Mg}\left(\mathrm{ClO}_{4}\right)_{2}$ [37], $\mathrm{ZrOCl} \cdot 5 \mathrm{H}_{2} \mathrm{O}$ [38], $\mathrm{Al}\left(\mathrm{H}_{2} \mathrm{PO}_{4}\right)$ [39], $\mathrm{BiCl}_{3}$ [40], $\mathrm{FeCl}_{3}$ [41], $\mathrm{YbCl}_{3}$ [42], $\mathrm{In}(\mathrm{OTf})_{3}$ [43], $\mathrm{Ce}(\mathrm{OTf})_{4}$ [44], $\mathrm{Al}(\mathrm{OTf})_{3}$ [45], $\mathrm{CAN}$ [46], $\mathrm{TaCl}_{5}-\mathrm{SiO}_{2}$ [47], and $\mathrm{SmI}_{2}$ [48] solid acids (montmorillonite KSF, silica sulfuric acid, Amberlyst-15, and Amberlite-IR 120) [49], base catalysts such as $\mathrm{CaCl}_{2}$ and $\mathrm{PPh}_{3}$ and other catalysts such as $\mathrm{ZnO}, \mathrm{TiO}_{2}$, tosyl chloride, and mesoporous aluminosilicate nanocage [50] have also been used to promote this reaction. Due to the above-mentioned factors, in this paper we reported the synthesis of $\alpha$-aminophosphonates with high yield using a recyclable catalyst for applications in medicine and industry.

\section{Results and Discussion}

In the initial experiments, the one-pot, three-component reaction of aniline, benzaldehyde, and diethyl phosphite was chosen as the model reaction to optimize the reaction conditions. In the present work, the procedures followed for the synthesis of $\alpha$-aminophosphonates are conventional reflux in toluene, in the presence of catalyst (Amberlite IRC-748) and microwave irradiation (solvent-free). The data obtained are 
TABLE 1: Reaction time and percentage yield of 4 in different reaction conditions.

\begin{tabular}{|c|c|c|c|c|c|}
\hline Entry & Catalyst used & Reaction condition & Reaction time & $\%$ yield & Reference \\
\hline 1 & $\mathrm{Al}\left(\mathrm{H}_{2} \mathrm{PO}_{4}\right)_{3}$ & Solvent-free $/ 100^{\circ} \mathrm{C}$ & $90 \mathrm{~min}$ & 93 & [39] \\
\hline 2 & $\mathrm{InCl}_{3}$ & THF/RT & $11 \mathrm{~h}$ & 92 & {$[34]$} \\
\hline 3 & $\mathrm{BiCl}_{3}$ & $\mathrm{CH}_{3} \mathrm{CN} /$ reflux & $6 \mathrm{~h}$ & 92 & {$[40]$} \\
\hline 4 & $\mathrm{FeCl}_{3}$ & $\mathrm{THF} / 60^{\circ} \mathrm{C}$ & $0.75 \mathrm{~h}$ & 92 & {$[41]$} \\
\hline 5 & $\mathrm{YbCl}_{3}$ & $\mathrm{CH}_{3} \mathrm{CN} / \mathrm{RT}$ & $24 \mathrm{~h}$ & 93 & {$[42]$} \\
\hline 6 & $\operatorname{In}(\mathrm{OTf})_{3}$ & THF/reflux & $21 \mathrm{~h}$ & 79 & {$[43]$} \\
\hline 7 & $\mathrm{Ce}(\mathrm{OTf})_{4}$ & Solvent-free $/ 50^{\circ} \mathrm{C}$ & $20 \mathrm{~min}$ & 94 & {$[44]$} \\
\hline 8 & $\mathrm{Mg}\left(\mathrm{ClO}_{4}\right)_{2}$ & Solvent-free $/ 80^{\circ} \mathrm{C}$ & $5 \mathrm{~h}$ & 99 & {$[37]$} \\
\hline 9 & CAN & Solvent-free/reflux & $30 \mathrm{~min}$ & 96 & {$[46]$} \\
\hline 10 & $\mathrm{TaCl}_{5}-\mathrm{SiO}_{2}$ & $\mathrm{CH}_{2} \mathrm{Cl}_{2} / \mathrm{RT}$ & $22 \mathrm{~h}$ & 92 & {$[47]$} \\
\hline 11 & $\mathrm{TiO}_{2}$ & Solvent-free $/ 50^{\circ} \mathrm{C}$ & $3.5 \mathrm{~h}$ & 98 & {$[50]$} \\
\hline 12 & $\mathrm{ZnO}$ & Solvent-free/RT & $9 \mathrm{~h}$ & 90 & {$[50]$} \\
\hline 13 & NBS & Solvent-free $/ 50^{\circ} \mathrm{C}$ & $3 \mathrm{~h}$ & 99 & {$[50]$} \\
\hline 14 & Silica sulfuric acid & $\mathrm{CH}_{3} \mathrm{CN} / \mathrm{RT}$ & $5 \mathrm{~h}$ & 87 & [49] \\
\hline 15 & 3D mesoporous aluminosilicate nanocage & $\mathrm{CH}_{3} \mathrm{CN} / 80^{\circ} \mathrm{C}$ & $4 \mathrm{~h}$ & 86 & {$[50]$} \\
\hline 16 & $\mathrm{Cu}(3,4$-tmtppa $)\left(\mathrm{MeSO}_{4}\right)_{4}$ & $\mathrm{H}_{2} \mathrm{O} / 80^{\circ} \mathrm{C}$ & $0.5 \mathrm{~h}$ & 96 & {$[50]$} \\
\hline 17 & $\mathrm{~B}-\mathrm{CD}$ & $\mathrm{H}_{2} \mathrm{O} /$ reflux & $24 \mathrm{~h}$ & 61 & {$[50]$} \\
\hline 18 & $\mathrm{CaCl}_{2}$ & Solvent-free $/ 60^{\circ} \mathrm{C}$ & $3 \mathrm{~h}$ & 90 & {$[48]$} \\
\hline 19 & $\mathrm{PPh}_{3}$ & Solvent-free $/ 60^{\circ} \mathrm{C}$ & $1 \mathrm{~h}$ & 87 & {$[48]$} \\
\hline 20 & $\mathrm{NbCl}_{5}$ & Solvent-free $/ 50^{\circ} \mathrm{C}$ & $30 \mathrm{~min}$ & 95 & {$[50]$} \\
\hline 21 & - & Toluene/reflux & $5 \mathrm{~h}$ & 81 & Present work \\
\hline 22 & - & Solvent-free/mw & $1 \mathrm{~min}$ & 87 & Present work \\
\hline 23 & Amberlyst-IRC 748 & Toluene/reflux & $30 \mathrm{~min}$ & 93 & Present work \\
\hline
\end{tabular}

shown in Table 1, entries 21-23. A comparison of the catalysts used in the Kabachnik-Field reaction for the synthesis of 4 is listed in Table 1, serial numbers 1-20.

The products $\alpha$-aminophosphonates were obtained by solvent-free microwave irradiation of aldehyde, amine, and diethyl phosphite for $1 \mathrm{~min}$. In toluene, without any catalyst, the product formed was in a good yield, but the time taken was 4 to $5 \mathrm{~h}$, which is considerably longer. Therefore, the reaction time has been reduced to $30 \mathrm{~min}$ by using Amberlite IRC-748 a recyclable catalyst. This catalyst is mildly acidic with iminodiacetic acid functional group. Amberlite IRC748 acts as an efficient and recyclable acidic promoter which yields good results when compared to the catalysts reported earlier (Table 1). The reaction mechanism proceeds as in case of acid catalysts. In optimization of reaction time, the yield of the product did not increase, when more than $5 \mathrm{mg}$ of catalyst was used. This suggested the use of $5 \mathrm{mg}$ of Amberlite catalyst for $0.005 \mathrm{~mol}$ of reactants. Thin layer chromatography (TLC) was employed to monitor reaction progress and to determine the purity of the products.

New $\alpha$-aminophosphonic acid esters (4a-o) were synthesized by a one-pot reaction using equimolar quantities of different substituted aromatic amines and aldehydes with diethylphosphite (Scheme 2). The reaction was carried out using catalytic amount of Amberlite IRC-748, in toluene for $30 \mathrm{~min}$. All the title compounds are readily soluble in polar organic solvents.

The IR spectra of compounds (4a-o) showed the NH band in the range of $3338-3438 \mathrm{~cm}^{-1}$. The sharp band observed in the range $1240-1291 \mathrm{~cm}^{-1}$ is due to the $\nu_{\mathrm{P}=\mathrm{O}}$, and a band for $\mathrm{P}-\mathrm{C}$ stretching occurred in the range $740-770 \mathrm{~cm}^{-1}$. All the stretching frequencies are compiled in Table 2. The ${ }^{1} \mathrm{H}$ NMR spectra of the compounds $(\mathbf{4 a - 0})$ were recorded in the DMSO- $\mathrm{d}_{6}$ solvent. The aromatic protons of $\alpha$-aminophosphonic acid esters appeared as a multiplet in the region $\delta$ 6.15-8.69. The $\mathrm{P}-\mathrm{C}-\mathrm{H}$ group proton resonated as a multiplet in the range $\delta 3.77-4.86$ due to coupling with phosphorus and $\mathrm{N}-\mathrm{H}$. The $\mathrm{N}-\mathrm{H}$ proton signal appeared at $\delta 4.58-5.90$ as a multiplet. The protons of $\mathrm{P}-\mathrm{O}-\mathrm{CH}_{2}-\mathrm{C}$ that appeared as a quartet at $\delta 3.56-3.62$ and $\mathrm{P}-\mathrm{O}-\mathrm{C}-\mathrm{CH}_{3}$ gave a triplet at $\delta$ 1.12-1.19. The compounds were analyzed by mass spectroscopy, the $\mathrm{M}+1$ peak confirmed product formation, and compounds containing one chlorine atom showed molecular ion peaks in a $3: 1$ ratio.

Antibacterial activity was carried out by the well diffusion method using nutrient agar medium, DMSO as control, and chloramphenicol as a standard bactericide. The antifungal activity was carried out by well diffusion method using potato dextrose agar (PDA) medium, DMSO as control, and fluconazole as a standard fungicide [51-54]. The antioxidant activity of the synthesized derivatives was evaluated using the $\mathrm{DPPH}$ (diphenyl picrylhydrazyl) radical scavenging assay by standard methods [55].

2.1. Antimicrobial Studies. The synthesized compounds (4ao) were screened for the antimicrobial activity. Most of the synthesized compounds showed inhibited growth of the strains (Table 3). Among the samples tested, $\mathbf{4 b}, \mathbf{4 d}, \mathbf{4 e}, \mathbf{4 i}$, 
TABLE 2: Elemental analysis and IR data of compounds (4a-o).

\begin{tabular}{|c|c|c|c|c|c|c|c|}
\hline \multirow{2}{*}{\multicolumn{2}{|c|}{ Mol. formula }} & \multicolumn{3}{|c|}{ Elemental analysis found (Calc.) } & \multicolumn{3}{|c|}{ IR spectral data in $\mathrm{cm}^{-1}$} \\
\hline & & $\mathrm{C}$ & $\mathrm{H}$ & $\mathrm{N}$ & $-\mathrm{NH}$ & $\mathrm{P}=\mathrm{O}$ & $\mathrm{P}-\mathrm{C}$ \\
\hline $4 a$ & $\mathrm{C}_{19} \mathrm{H}_{25} \mathrm{ClNO}_{5} \mathrm{P}$ & $55.23(55.14)$ & $6.02(6.09)$ & $3.32(3.38)$ & 3390 & 1285 & 747 \\
\hline $4 b$ & $\mathrm{C}_{17} \mathrm{H}_{20} \mathrm{ClN}_{2} \mathrm{O}_{7} \mathrm{P}$ & $47.38(47.40)$ & $4.59(4.68)$ & $6.47(6.50)$ & 3338 & 1241 & 739 \\
\hline $4 c$ & $\mathrm{C}_{18} \mathrm{H}_{22} \mathrm{ClN}_{2} \mathrm{O}_{7} \mathrm{P}$ & $48.57(48.60)$ & $4.94(4.99)$ & $6.27(6.30)$ & 3135 & 1210 & 751 \\
\hline $4 d$ & $\mathrm{C}_{19} \mathrm{H}_{25} \mathrm{ClNO}_{5} \mathrm{P}$ & $55.18(55.14)$ & $6.13(6.09)$ & $3.32(3.38)$ & 3381 & 1268 & 745 \\
\hline $4 e$ & $\mathrm{C}_{17} \mathrm{H}_{20} \mathrm{ClN}_{2} \mathrm{O}_{7} \mathrm{P}$ & $47.37(47.40)$ & $4.69(4.68)$ & $6.48(6.50)$ & 3340 & 1263 & 765 \\
\hline $4 \mathrm{f}$ & $\mathrm{C}_{18} \mathrm{H}_{22} \mathrm{ClN}_{2} \mathrm{O}_{7} \mathrm{P}$ & $48.58(48.60)$ & $4.94(4.99)$ & $6.27(6.30)$ & 3421 & 1266 & 756 \\
\hline $4 \mathrm{~g}$ & $\mathrm{C}_{20} \mathrm{H}_{28} \mathrm{NO}_{5} \mathrm{P}$ & $61.14(61.06)$ & $7.13(7.17)$ & $3.52(3.56)$ & 3405 & 1276 & 743 \\
\hline $4 h$ & $\mathrm{C}_{18} \mathrm{H}_{23} \mathrm{~N}_{2} \mathrm{O}_{7} \mathrm{P}$ & $52.72(52.68)$ & $5.68(5.65)$ & $6.80(6.83)$ & 3401 & 1271 & 741 \\
\hline $4 \mathbf{i}$ & $\mathrm{C}_{19} \mathrm{H}_{25} \mathrm{~N}_{2} \mathrm{O}_{7} \mathrm{P}$ & $53.75(53.77)$ & $5.97(5.94)$ & $6.54(6.60)$ & 3375 & 1256 & 762 \\
\hline $4 j$ & $\mathrm{C}_{16} \mathrm{H}_{28} \mathrm{NO}_{5} \mathrm{P}$ & $55.61(55.64)$ & $8.19(8.17)$ & $3.99(4.06)$ & 3399 & 1235 & 748 \\
\hline $4 \mathbf{k}$ & $\mathrm{C}_{14} \mathrm{H}_{23} \mathrm{~N}_{2} \mathrm{O}_{7} \mathrm{P}$ & 46.45 (46.41) & $6.44(6.40)$ & $7.70(7.73)$ & 3410 & 1247 & 753 \\
\hline 41 & $\mathrm{C}_{15} \mathrm{H}_{25} \mathrm{~N}_{2} \mathrm{O}_{7} \mathrm{P}$ & $47.84(47.87)$ & $6.73(6.70)$ & $7.39(7.44)$ & 3438 & 1278 & 769 \\
\hline $4 \mathrm{~m}$ & $\mathrm{C}_{19} \mathrm{H}_{25} \mathrm{ClNO}_{5} \mathrm{P}$ & $55.18(55.14)$ & $6.12(6.09)$ & $3.35(3.38)$ & 3385 & 1290 & 758 \\
\hline $4 n$ & $\mathrm{C}_{17} \mathrm{H}_{20} \mathrm{ClN}_{2} \mathrm{O}_{7} \mathrm{P}$ & $47.46(47.40)$ & $4.63(4.68)$ & $6.47(6.50)$ & 3356 & 1274 & 749 \\
\hline 40 & $\mathrm{C}_{18} \mathrm{H}_{22} \mathrm{ClN}_{2} \mathrm{O}_{7} \mathrm{P}$ & $48.63(48.60)$ & $4.97(4.99)$ & $6.26(6.30)$ & 3391 & 1256 & 758 \\
\hline
\end{tabular}

TABLE 3: Antimicrobial studies.

\begin{tabular}{|c|c|c|c|c|c|c|c|c|}
\hline \multirow{3}{*}{ Entry } & \multirow{3}{*}{ Comp. } & \multicolumn{7}{|c|}{ Zone of inhibition in $\mathrm{mm}$} \\
\hline & & \multicolumn{5}{|c|}{ Antibacterial } & \multicolumn{2}{|c|}{ Antifungal } \\
\hline & & S. aureus & B. subtilis & S. typhi & E. coli & S. cocus & C. albicans & A. niger \\
\hline 1 & $4 \mathrm{a}$ & 02 & 06 & 09 & 05 & 07 & 07 & 11 \\
\hline 2 & $4 \mathrm{~b}$ & 08 & 07 & 06 & 12 & 11 & 08 & 10 \\
\hline 3 & $4 c$ & 05 & 06 & 06 & 07 & 05 & 07 & 06 \\
\hline 4 & $4 \mathrm{~d}$ & 05 & 09 & 06 & 11 & 10 & 09 & 09 \\
\hline 5 & $4 \mathrm{e}$ & 09 & 10 & 07 & 06 & 11 & 10 & 11 \\
\hline 6 & $4 \mathrm{f}$ & 09 & 10 & 08 & 12 & 08 & 09 & 11 \\
\hline 7 & $4 \mathrm{~g}$ & 08 & 08 & 09 & 10 & 08 & 09 & 09 \\
\hline 8 & $4 \mathrm{~h}$ & 05 & 06 & 06 & 07 & 05 & 07 & 06 \\
\hline 9 & $4 \mathrm{i}$ & 05 & 09 & 06 & 11 & 10 & 09 & 09 \\
\hline 10 & $4 j$ & 09 & 10 & 07 & 06 & 11 & 10 & 11 \\
\hline 11 & $4 \mathrm{k}$ & 10 & 13 & 08 & 12 & 11 & 11 & 09 \\
\hline 12 & 41 & 08 & 11 & 09 & 10 & 08 & 10 & 12 \\
\hline 13 & $4 \mathrm{~m}$ & 08 & 10 & 06 & 11 & 05 & 08 & 06 \\
\hline 14 & $4 n$ & 07 & 05 & 07 & 08 & 07 & 05 & 06 \\
\hline 15 & $4 \mathrm{o}$ & 09 & 12 & 11 & 12 & 08 & 10 & 09 \\
\hline 16 & Std1. & 08 & 07 & 10 & 10 & 09 & - & - \\
\hline 17 & Std2. & - & - & - & - & - & 08 & 09 \\
\hline 18 & Cont. & - & - & - & - & - & - & - \\
\hline
\end{tabular}

Where Std1. is chloramphenicol, Std2. is fluconazole, and Cont. is DMSO.

and $\mathbf{4} \mathbf{j}$ showed promising activity against most of the stains compared to the standard drug used. The presence of substitutions like $-\mathrm{OH},-\mathrm{Cl}$, and $-\mathrm{NO}_{2}$ enabled the compounds to show promising activity.

2.2. Antioxidant Activity. The novel compounds were checked for the free radical scavenging activity by the DPPH method, and the data are listed in Table 4. The graphical representation of the DPPH activity, indicated in Figure 1, showed that most of the compounds are good antioxidants with more than $50 \%$ scavenging activity. Among them, the compounds $4 \mathbf{c}, \mathbf{4 e}, \mathbf{4 h}$, and $\mathbf{4} \mathbf{j}$ showed higher activity than the standard used. This may be attributed to the presence of substitutions like $-\mathrm{NO}_{2}$ and $-\mathrm{OH}$ groups in the compounds synthesized.

2.3. Experimental Procedure. All the reagents and solvents were used as received from commercial suppliers, unless otherwise stated. All chemicals used for the synthesis were of analytical grade or laboratory grade and purchased from HiMedia Laboratories Pvt. Ltd., Sigma Chemical Co., USA, 
TABLE 4: Antioxidant activity.

\begin{tabular}{|c|c|c|c|c|c|}
\hline \multirow{2}{*}{ Compounds } & \multicolumn{5}{|c|}{$\%$ Scavenging activity at different concentrations } \\
\hline & $25 \mu \mathrm{g} / \mathrm{mL}$ & $50 \mu \mathrm{g} / \mathrm{mL}$ & $100 \mu \mathrm{g} / \mathrm{mL}$ & $250 \mu \mathrm{g} / \mathrm{mL}$ & $500 \mu \mathrm{g} / \mathrm{mL}$ \\
\hline $4 \mathbf{a}$ & 12.32 & 24.15 & 54.87 & 72.03 & 84.26 \\
\hline $4 b$ & 7.05 & 14.25 & 23.57 & 42.1 & 66.32 \\
\hline $4 c$ & 24.15 & 40.25 & 60.58 & 87.51 & 98.35 \\
\hline $4 d$ & 7.61 & 17.25 & 29.45 & 39.25 & 61.23 \\
\hline $4 e$ & 20.15 & 32.45 & 63.25 & 88.71 & 95.26 \\
\hline 4f & 12.89 & 20.45 & 29.87 & 38.26 & 42.1 \\
\hline $4 g$ & 12.32 & 24.15 & 54.87 & 72.03 & 84.26 \\
\hline $4 h$ & 24.15 & 40.25 & 60.58 & 87.51 & 98.35 \\
\hline $4 i$ & 7.61 & 17.25 & 29.45 & 39.25 & 61.23 \\
\hline $4 j$ & 20.15 & 32.45 & 63.25 & 88.71 & 95.26 \\
\hline $4 k$ & 12.86 & 21.56 & 32.79 & 42.13 & 56.52 \\
\hline 41 & 10.23 & 22.83 & 33.56 & 51.23 & 62.81 \\
\hline $4 \mathrm{~m}$ & 6.32 & 12.52 & 21.83 & 34.54 & 49.42 \\
\hline $4 n$ & 3.25 & 11.59 & 20.94 & 33.54 & 46.23 \\
\hline 40 & 6.23 & 11.25 & 20.38 & 32.45 & 48.24 \\
\hline BHT & 12.35 & 25.72 & 58.51 & 86.25 & 94.32 \\
\hline
\end{tabular}

BHT: butylated hydroxytoluene.

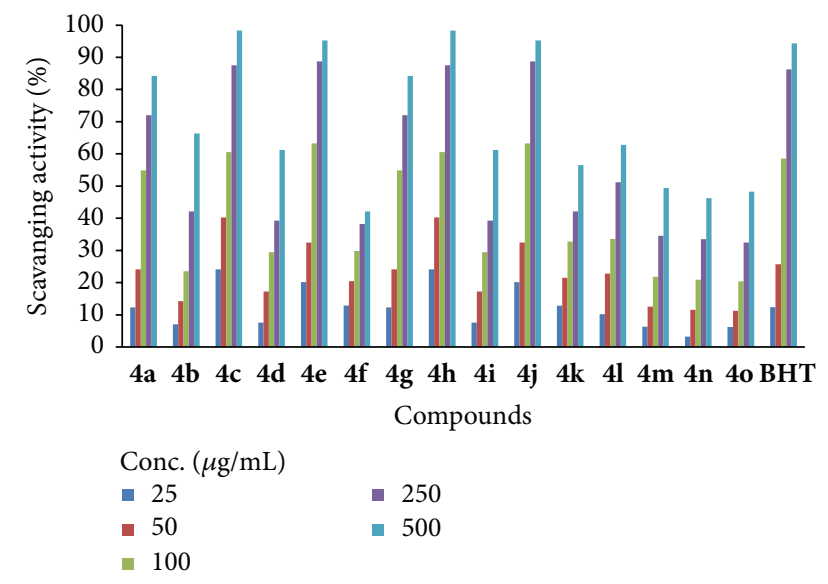

FIGURE 1: DPPH radical scavenging activity of the synthesized compounds.

E. Merck, Germany, and Sarabhai Merck Company, India, and specialty chemicals are procured as samples from the commercial suppliers in India. Mass spectra of the synthesized compounds were recorded on Agilent 6320 Ion Trap mass spectrometer. IR spectra were recorded on a Shimadzu IR-470 spectrometer. ${ }^{1} \mathrm{H}$ NMR spectra were recorded on a Bruker DRX-300 Avance spectrometer (300 MHz).

\subsection{General Procedure for the Synthesis (Scheme 1)}

2.4.1. Synthetic Procedure-a. A mixture of benzaldehyde $(0.005 \mathrm{~mol})$, aniline $(0.005 \mathrm{~mol})$ and diethylphosphite $(0.005 \mathrm{~mol})$ in dry toluene was stirred for $10 \mathrm{~min}$ at room temperature. Then the temperature was raised to reflux for $5 \mathrm{~h}$. The reaction was monitored by TLC. After completion of the reaction, toluene was removed by distillation and the residue was purified using column chromatography $(6: 4$, ethyl acetate: hexane).

2.4.2. Synthetic Procedure $\boldsymbol{b}$. A mixture of benzaldehyde $(0.005 \mathrm{~mol})$, aniline $(0.005 \mathrm{~mol})$, diethylphosphite $(0.005 \mathrm{~mol})$, and $5 \mathrm{mg}$ of Amberlite IRC-748 in dry toluene was stirred for $10 \mathrm{~min}$ at room temperature. Then it was refluxed for $30 \mathrm{~min}$. The reaction was monitored by TLC. After completion of the reaction, the mixture was filtered to separate the solid catalyst. The filtrate was distilled to remove toluene, and the residue obtained was purified using column chromatography $(6: 4$, ethyl acetate: hexane).

2.4.3. Synthetic Procedure c. A mixture of benzaldehyde $(0.005 \mathrm{~mol})$, aniline $(0.005 \mathrm{~mol})$, and diethylphosphite $(0.005 \mathrm{~mol})$ was irradiated with microwaves twice, for $30 \mathrm{sec}$, to control the temperature. The reaction was monitored by TLC. After completion of the reaction, the crude product was purified using column chromatography $(6: 4$, ethyl acetate: hexane).

2.4.4. Synthesis of Compounds (4a-o). The compounds (4ao) (Scheme 2) were synthesized by following the aforementioned synthetic procedure $b$.

Diethyl(4-chlorophenylamino)(3-ethoxy-4-hydroxyphenyl)methylphosphonate (4a). Yield-92.1\%, colour-dark yellow. ${ }^{1} \mathrm{H}-\mathrm{NMR}\left(300 \mathrm{MHz}, \mathrm{DMSO}-\mathrm{d}_{6}\right) \delta 10.1(\mathrm{~s}, 1 \mathrm{H},-\mathrm{OH}), 7.73-$ $6.40(\mathrm{~m}, 7 \mathrm{H}, \mathrm{Ar}-\mathrm{H}), 5.38(\mathrm{~m}, 1 \mathrm{H}, \mathrm{N}-\mathrm{H}), 3.98(\mathrm{~m}, 1 \mathrm{H}, \mathrm{P}-\mathrm{CH})$, $3.71\left(\mathrm{q}, 6 \mathrm{H},-\mathrm{OCH}_{2}\right), 1.32\left(\mathrm{t}, 9 \mathrm{H}, \mathrm{O}-\mathrm{CCH}_{3}\right) .{ }^{31} \mathrm{P}-\mathrm{NMR}$ $\left(161.9 \mathrm{MHz}\right.$, DMSO- $\left.\mathrm{d}_{6}\right) \delta 32.5 . \mathrm{M} / z: 413$ and 415 with $3: 1$ ratio. M.P. $175-178^{\circ} \mathrm{C}$.

Diethyl(4-chlorophenylamino)(3,4-dihydroxy-5-nitrophenyl)methylphosphonate (4b). Yield-79.4\%, colour-brown, 
<smiles>O=Cc1ccccc1</smiles><smiles>Nc1ccccc1</smiles>

2

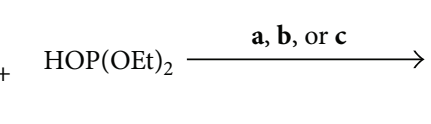<smiles>CCOP(=O)(OCC)C(Nc1ccccc1)c1ccccc1</smiles>

4

a: conventional method: toluene-reflux, 4-5 h.

b: catalytic amount of Amberlite-IRC 748, toluene, $30 \mathrm{~min}$.

c: microwave irradiation. 30 to $90 \mathrm{~s}$.

Scheme 1: Kabachnik-Fields reaction.

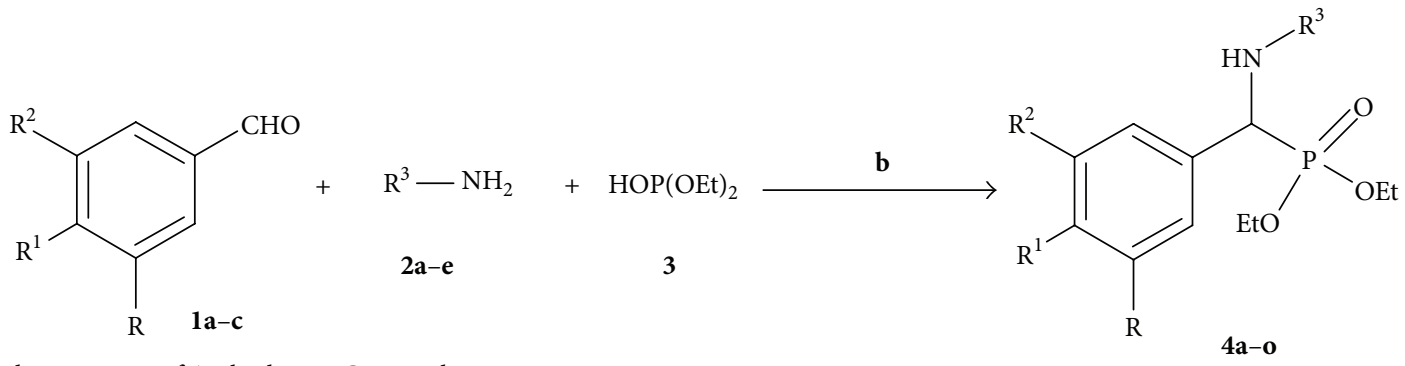

b: catalytic amount of Amberlyst-IRC 748, toluene, $30 \mathrm{~min}$.

\begin{tabular}{c|ccc}
$\mathbf{1}$ & $\mathrm{R}$ & $\mathrm{R}^{1}$ & $\mathrm{R}^{2}$ \\
\hline $\mathbf{a}$ & $-\mathrm{OEt}$ & $-\mathrm{OH}$ & $-\mathrm{H}$ \\
$\mathbf{b}$ & $-\mathrm{OH}$ & $-\mathrm{OH}$ & $-\mathrm{NO}_{2}$ \\
$\mathbf{c}$ & $-\mathrm{OMe}$ & $-\mathrm{OH}$ & $-\mathrm{NO}_{2}$
\end{tabular}

\begin{tabular}{c|c}
$\mathbf{2}$ & $\mathrm{R}^{3}$ \\
\hline $\mathbf{a}$ & $4-\mathrm{ClC}_{6} \mathrm{H}_{4}-$ \\
$\mathbf{b}$ & $3-\mathrm{ClC}_{6} \mathrm{H}_{4}-$ \\
$\mathbf{c}$ & $\mathrm{C}_{6} \mathrm{H}_{5} \mathrm{CH}_{2-}$ \\
d & $n-\mathrm{C}_{3} \mathrm{H}_{7^{-}}$ \\
e & $2-\mathrm{ClC}_{6} \mathrm{H}_{4-}$
\end{tabular}

\begin{tabular}{c|cccc}
$\mathbf{4}$ & $\mathrm{R}$ & $\mathrm{R}^{1}$ & $\mathrm{R}^{2}$ & $\mathrm{R}^{3}$ \\
\hline $\mathbf{4 a}$ & $-\mathrm{OEt}$ & $-\mathrm{OH}$ & $-\mathrm{H}$ & $4-\mathrm{ClC}_{6} \mathrm{H}_{4}-$ \\
$\mathbf{4 b}$ & $-\mathrm{OH}$ & $-\mathrm{OH}$ & $-\mathrm{NO}_{2}$ & $4-\mathrm{ClC}_{6} \mathrm{H}_{4}-$ \\
$\mathbf{4}$ & $-\mathrm{OMe}$ & $-\mathrm{OH}$ & $-\mathrm{NO}_{2}$ & $4-\mathrm{ClC}_{6} \mathrm{H}_{4}-$ \\
$\mathbf{4 d}$ & $-\mathrm{OEt}$ & $-\mathrm{OH}$ & $-\mathrm{H}$ & $3-\mathrm{ClC}_{6} \mathrm{H}_{4}-$ \\
$\mathbf{4}$ & $-\mathrm{OH}$ & $-\mathrm{OH}$ & $-\mathrm{NO}_{2}$ & $3-\mathrm{ClC}_{6} \mathrm{H}_{4}-$ \\
$\mathbf{4 f}$ & $-\mathrm{OMe}$ & $-\mathrm{OH}$ & $-\mathrm{NO}_{2}$ & $3-\mathrm{ClC}_{6} \mathrm{H}_{4}-$ \\
$\mathbf{4 g}$ & $-\mathrm{OEt}$ & $-\mathrm{OH}$ & $-\mathrm{H}$ & $\mathrm{C}_{6} \mathrm{H}_{5} \mathrm{CH}_{2}-$ \\
$\mathbf{4 h}$ & $-\mathrm{OH}$ & $-\mathrm{OH}$ & $-\mathrm{NO}_{2}$ & $\mathrm{C}_{6} \mathrm{H}_{5} \mathrm{CH}_{2}-$ \\
$\mathbf{4 i}$ & $-\mathrm{OMe}$ & $-\mathrm{OH}$ & $-\mathrm{NO}_{2}$ & $\mathrm{C}_{6} \mathrm{H}_{5} \mathrm{CH}_{2}-$ \\
$\mathbf{4 j}$ & $-\mathrm{OEt}$ & $-\mathrm{OH}$ & $-\mathrm{H}$ & $n-\mathrm{C}_{3} \mathrm{H}_{7^{-}}$ \\
$\mathbf{4 k}$ & $-\mathrm{OH}$ & $-\mathrm{OH}$ & $-\mathrm{NO}_{2}$ & $n-\mathrm{C}_{3} \mathrm{H}_{7^{-}}$ \\
$\mathbf{4 l}$ & $-\mathrm{OMe}$ & $-\mathrm{OH}$ & $-\mathrm{NO}_{2}$ & $n-\mathrm{C}_{3} \mathrm{H}_{7}-$ \\
$\mathbf{4 m}$ & $-\mathrm{OEt}$ & $-\mathrm{OH}$ & $-\mathrm{H}$ & $2-\mathrm{ClC}_{6} \mathrm{H}_{4}-$ \\
$\mathbf{4 n}$ & $-\mathrm{OH}$ & $-\mathrm{OH}$ & $-\mathrm{NO}_{2}$ & $2-\mathrm{ClC}_{6} \mathrm{H}_{4}-$ \\
$\mathbf{4 o}$ & $-\mathrm{OMe}$ & $-\mathrm{OH}$ & $-\mathrm{NO}_{2}$ & $2-\mathrm{ClC}_{6} \mathrm{H}_{4}-$
\end{tabular}

Scheme 2: Newly synthesized derivatives.

${ }^{1} \mathrm{H}-\mathrm{NMR}\left(300 \mathrm{MHz}\right.$, DMSO-d $\left.\mathrm{d}_{6}\right) \delta 10.5$ (br, $\left.2 \mathrm{H},-\mathrm{OH}\right), 7.90-$ $6.79(\mathrm{~m}, 6 \mathrm{H}, \mathrm{Ar}-\mathrm{H}), 5.45(\mathrm{~m}, 1 \mathrm{H}, \mathrm{N}-\mathrm{H}), 4.14\left(\mathrm{~m}, 1 \mathrm{H}, \mathrm{P}-\mathrm{CH}_{2}\right)$, $3.68\left(\mathrm{q}, 4 \mathrm{H}, \mathrm{P}-\mathrm{OCH}_{2}\right), 1.25\left(\mathrm{t}, 6 \mathrm{H}, \mathrm{P}-\mathrm{CCH}_{3}\right) .{ }^{31} \mathrm{P}-\mathrm{NMR}$ $\left(161.9 \mathrm{MHz}, \mathrm{DMSO}-\mathrm{d}_{6}\right) \delta 31.6 . \mathrm{M} / z: 430$ and 432 with $3: 1$ ratio. M.P. $178-181^{\circ} \mathrm{C}$.

Diethyl(4-chlorophenylamino)(4-hydroxy-3-methoxy-5-nitrophenyl)methylphosphonate (4c). Yield-86.0\%, colour-brown. $1 \mathrm{H}-\mathrm{NMR}\left(300 \mathrm{MHz}, \mathrm{DMSO}_{-} \mathrm{d}_{6}\right) \delta 10.5(\mathrm{~s}, 1 \mathrm{H},-\mathrm{OH}), \delta$
8.22-6.52 (m, 6H, Ar-H), $5.44(\mathrm{~m}, 1 \mathrm{H}, \mathrm{N}-\mathrm{H}), 4.15(\mathrm{~m}, 1 \mathrm{H}$, $\mathrm{P}-\mathrm{CH}), 3.64\left(\mathrm{q}, 4 \mathrm{H},-\mathrm{OCH}_{2}\right), 2.95\left(\mathrm{~s}, 3 \mathrm{H},-\mathrm{OCCH}_{3}\right), 1.04(\mathrm{t}$, $\left.6 \mathrm{H},-\mathrm{OCCH}_{3}\right) .{ }^{31} \mathrm{P}-\mathrm{NMR}\left(161.9 \mathrm{MHz}, \mathrm{DMSO}-\mathrm{d}_{6}\right) \delta 31.5 . \mathrm{M} / z$ : 444 and 446 with $3: 1$ ratio. M.P. $162-164^{\circ} \mathrm{C}$.

Diethyl(3-chlorophenylamino)(3-ethoxy-4-hydroxyphenyl)methylphosphonate (4d). Yield-93.4\%, colour-yellow. ${ }^{1} \mathrm{H}$ NMR $\left(300 \mathrm{MHz}, \mathrm{DMSO}-\mathrm{d}_{6}\right) \delta 10.3(\mathrm{~s}, 1 \mathrm{H},-\mathrm{OH}), 8.21-6.89$ $(\mathrm{m}, 7 \mathrm{H}, \operatorname{Ar}-\mathrm{H}), 5.68(\mathrm{~m}, 1 \mathrm{H}, \mathrm{N}-\mathrm{H}), 4.20(\mathrm{~m}, 1 \mathrm{H}, \mathrm{P}-\mathrm{CH})$, 
$3.82\left(\mathrm{q}, 6 \mathrm{H},-\mathrm{OCH}_{2}\right), 1.18\left(\mathrm{t}, 9 \mathrm{H},-\mathrm{CCH}_{3}\right) .{ }^{31} \mathrm{P}-\mathrm{NMR}(161.9 \mathrm{MHz}$, DMSO- $\left.\mathrm{d}_{6}\right) \delta 32.6 . \mathrm{M} / z$ : 413 and 415 with $3: 1$ ratio. M.P. 167$169^{\circ} \mathrm{C}$.

Diethyl(3-chlorophenylamino)(3,4-dihydroxy-5-nitrophenyl)methylphosphonate (4e). Yield-82.9\%, colour-brown. ${ }^{1} \mathrm{H}-\mathrm{NMR}\left(300 \mathrm{M} \mathrm{Hz}, \mathrm{DMSO}-\mathrm{d}_{6}\right) \delta 10.4$ (br, 2H, -OH), 8.11$6.68(\mathrm{~m}, 6 \mathrm{H}, \mathrm{Ar}-\mathrm{H}), 5.55(\mathrm{~m}, 1 \mathrm{H}, \mathrm{N}-\mathrm{H}), 3.92(\mathrm{~m}, 1 \mathrm{H}, \mathrm{P}-\mathrm{CH})$, $3.81\left(\mathrm{q}, 4 \mathrm{H}, \mathrm{P}-\mathrm{OCH}_{2}\right), 1.33\left(\mathrm{t}, 6 \mathrm{H},-\mathrm{CCH}_{3}\right) .{ }^{31} \mathrm{P}-\mathrm{NMR}$ $\left(161.9 \mathrm{MHz}, \mathrm{DMSO}-\mathrm{d}_{6}\right) \delta 30.6 . \mathrm{M} / z: 430$ and 432 with $3: 1$ ratio. M.P. $143-147^{\circ} \mathrm{C}$.

Diethyl(3-chlorophenylamino)(4-hydroxy-3-methoxy-5-nitrophenyl)methylphosphonate(4f). Yield-76.5\%, colour-dark brown, ${ }^{1} \mathrm{H}-\mathrm{NMR}\left(300 \mathrm{MHz}, \mathrm{DMSO}-\mathrm{d}_{6}\right) \delta 10.2(\mathrm{~s}, 1 \mathrm{H},-\mathrm{OH})$, 8.10-6.59 (m, 6H, Ar-H), 5.03 (m, 1H, N-H), 4.06 (m, 1H, P$\mathrm{CH}), 3.83\left(\mathrm{q}, 4 \mathrm{H}, \mathrm{P}-\mathrm{OCH}_{2}\right), 3.14\left(\mathrm{~s}, 3 \mathrm{H},-\mathrm{OCH}_{3}\right), 1.13(\mathrm{t}, 6 \mathrm{H}$, $\left.-\mathrm{OCCH}_{3}\right) .{ }^{31} \mathrm{P}-\mathrm{NMR}\left(161.9 \mathrm{MHz}, \mathrm{DMSO}-\mathrm{d}_{6}\right) \delta 31.6 . \mathrm{M} / z: 444$ and 446 with $3: 1$ ratio. M.P. $178-180^{\circ} \mathrm{C}$.

Diethyl(benzylamino)(3-ethoxy-4-hydroxyphenyl)methylphosphonate (4g). Yield-94.1\%, colour-pale yellow. ${ }^{1} \mathrm{H}-\mathrm{NMR}$ $\left(300 \mathrm{MHz}, \mathrm{DMSO}-\mathrm{d}_{6}\right) \delta 9.8(\mathrm{~s}, 1 \mathrm{H},-\mathrm{OH}), 8.05-6.59(\mathrm{~m}, 8 \mathrm{H}$, Ar-H), 4.87 (m, 1H, N-H), $4.32\left(\mathrm{~d}, 2 \mathrm{H}, \mathrm{N}-\mathrm{CH}_{2}\right), 4.13(\mathrm{~m}, 1 \mathrm{H}$, $\mathrm{P}-\mathrm{CH}), 3.77\left(\mathrm{q}, 6 \mathrm{H},-\mathrm{OCH}_{2}\right), 1.23\left(\mathrm{t}, 9 \mathrm{H},-\mathrm{CCH}_{3}\right) .{ }^{31} \mathrm{P}-\mathrm{NMR}$ (161.9 MHz, DMSO-d ${ }_{6}$ ) $\delta 32.6 . M / z: 394$. M.P. $134-136^{\circ} \mathrm{C}$.

Diethyl(benzylamino)(3,4-dihydroxy-5-nitrophenyl)methylphosphonate (4h). Yield-85.4\%, colour-reddish brown, ${ }^{1} \mathrm{H}-$ NMR (300 MHz, DMSO-d 6 ) $\delta 10.1$ (br, 2H, -OH), 7.92-6.62 (m, 7H, Ar-H), $4.98(\mathrm{~m}, 1 \mathrm{H}, \mathrm{N}-\mathrm{H}), 4.37$ (d, 2H, N-CH $\left.{ }_{2}\right), 4.29$ (m, 1H, P-CH), 3.76 (q, 4H, P-OCH ${ }_{2}$ ), 1.28 (t, 6H, P-CCH ${ }_{3}$ ). ${ }^{31} \mathrm{P}-\mathrm{NMR}$ (161.9 MHz, DMSO-d $\left.{ }_{6}\right) \delta$ 29.4. M/z: 411. M.P. $123-125^{\circ} \mathrm{C}$.

Diethyl(benzylamino)(4-hydroxy-3-methoxy-5-nitrophenyl)methylphosphonate (4i). Yield-82.5\%, colour-dark brown ${ }^{1} \mathrm{H}-\mathrm{NMR}\left(300 \mathrm{MHz}, \mathrm{DMSO}-\mathrm{d}_{6}\right) \delta 10.2(\mathrm{~s}, 1 \mathrm{H},-\mathrm{OH})$, 8.26-6.69 (m, 7H, Ar-H), 4.78 (m, 1H, N-H), 4.32 (d, 2H, N$\mathrm{CH}_{2}$ ), 4.18 (m, 1H, P-CH), 3.81 (q, 4H, P-OCH ${ }_{2}$ ), 2.31 (s, 3H, $\left.-\mathrm{OCH}_{3}\right), 1.31\left(\mathrm{t}, 6 \mathrm{H}, \mathrm{P}-\mathrm{CCH}_{3}\right) .{ }^{31} \mathrm{P}-\mathrm{NMR} \quad(161.9 \mathrm{MHz}$, DMSO- $\left.\mathrm{d}_{6}\right) \delta 31.3 . \mathrm{M} / z: 425$. M.P. $138-140^{\circ} \mathrm{C}$.

Diethyl(3-ethoxy-4-hydroxyphenyl)(propylamino)methylphosphonate (4j). Yield-91.8\%, colour-dark yellow. ${ }^{1} \mathrm{H}-\mathrm{NMR}$ $\left(300 \mathrm{MHz}, \mathrm{DMSO}-\mathrm{d}_{6}\right) \delta 10.45(\mathrm{~s}, 1 \mathrm{H},-\mathrm{OH}), 7.93-6.89(\mathrm{~m}, 3 \mathrm{H}$, Ar-H), 4.67 (m, 1H, N-H), 4.25 (m, 1H, P-CH), $3.71(\mathrm{q}, 6 \mathrm{H}$, $-\mathrm{OCH}_{2}$ ), 3.06 (q, 2H, $\left.\mathrm{NCH}_{2} \mathrm{C}\right), 1.56\left(\mathrm{~m}, 2 \mathrm{H}, \mathrm{CCH}_{2} \mathrm{C}\right), 1.23(\mathrm{t}$, $\left.9 \mathrm{H},-\mathrm{OCCH}_{3}\right), 0.96\left(\mathrm{t}, 3 \mathrm{H},-\mathrm{CCCH}_{3}\right) .{ }^{31} \mathrm{P}-\mathrm{NMR}(161.9 \mathrm{MHz}$, DMSO $\left.-\mathrm{d}_{6}\right) \delta 32.9 . M / z: 346$. M.P. $121-123^{\circ} \mathrm{C}$.

Diethyl(3,4-dihydroxy-5-nitrophenyl)(propylamino)methylphosphonate (4k). Yield-87.5\%, colour-reddish brown. ${ }^{1} \mathrm{H}-$ NMR (300 MHz, DMSO-d $\left.{ }_{6}\right) \delta 10.25$ (br, 2H, -OH), 7.32-6.64 $(\mathrm{m}, 2 \mathrm{H}, \mathrm{Ar}-\mathrm{H}), 4.58(\mathrm{~m}, 1 \mathrm{H}, \mathrm{N}-\mathrm{H}), 4.28$ (m, 1H, P-CH), 3.73 (q, $\left.4 \mathrm{H}, \mathrm{P}-\mathrm{OCH}_{2}\right), 3.2\left(\mathrm{q}, 2 \mathrm{H}, \mathrm{NCH}_{2} \mathrm{C}\right), 1.83\left(\mathrm{~m}, 2 \mathrm{H}, \mathrm{CCH}_{2} \mathrm{C}\right)$, $1.09\left(\mathrm{t}, 3 \mathrm{H}, \mathrm{CCCH}_{3}\right), 1.02\left(\mathrm{t}, 6 \mathrm{H}, \mathrm{P}-\mathrm{CCH}_{3}\right) .{ }^{31} \mathrm{P}-\mathrm{NMR}$ (161.9 MHz, DMSO- $\mathrm{d}_{6}$ ) $\delta 28.9 . \mathrm{M} / z: 363$. M.P. $128-130^{\circ} \mathrm{C}$.
Diethyl(4-hydroxy-3-methoxy-5-nitrophenyl)(propylamino)methylphosphonate (41). Yield-82.4\%, colour-brown. ${ }^{1} \mathrm{H}$ NMR (300 MHz, DMSO-d d $_{6} \delta 10.35$ (s, 1H, -OH), 7.56-6.34 $(\mathrm{m}, 2 \mathrm{H}, \mathrm{Ar}-\mathrm{H}), 4.37(\mathrm{~m}, 1 \mathrm{H}, \mathrm{N}-\mathrm{H}), 4.17(\mathrm{~m}, 1 \mathrm{H}, \mathrm{P}-\mathrm{CH})$, 3.77 (q, 4H, P-OCH $\left.{ }_{2}\right), 3.12\left(\mathrm{q}, 2 \mathrm{H}, \mathrm{NCH}_{2} \mathrm{C}\right), 3.05$ (s, 6H, $\left.-\mathrm{OCH}_{3}\right), 1.69\left(\mathrm{~m}, 2 \mathrm{H}, \mathrm{CCH}_{2} \mathrm{C}\right), 1.24\left(\mathrm{t}, 6 \mathrm{H}, \mathrm{P}-\mathrm{CCH}_{3}\right), 1.02(\mathrm{t}$, $\left.3 \mathrm{H}, \mathrm{CCCH}_{3}\right) .{ }^{31} \mathrm{P}-\mathrm{NMR}\left(161.9 \mathrm{MHz}, \mathrm{DMSO}-\mathrm{d}_{6}\right) \delta 31.82 . \mathrm{M} / z$ : 377. M.P. $112-114^{\circ} \mathrm{C}$.

Diethyl(2-chlorophenylamino)(3-ethoxy-4-hydroxyphenyl)methylphosphonate (4m). Yield-93.2\%, colour-yellow. ${ }^{1} \mathrm{H}$ NMR (300 MHz, DMSO-d 6 ) $\delta 10.1(\mathrm{~s}, 1 \mathrm{H},-\mathrm{OH}), 8.15-6.78$ (m, 7H, Ar-H), 5.21 (m, 1H, N-H), 3.98 (m, 1H, P-CH), 3.72 $\left(\mathrm{q}, 6 \mathrm{H},-\mathrm{OCH}_{2}\right), 1.28\left(\mathrm{t}, 9 \mathrm{H},-\mathrm{CCH}_{3}\right) .{ }^{31} \mathrm{P}-\mathrm{NMR}(161.9 \mathrm{MHz}$, DMSO- $\left.\mathrm{d}_{6}\right) \delta 32.7 . \mathrm{M} / z$ : 413 and 415 with $3: 1$ ratio. M.P. $182-$ $184^{\circ} \mathrm{C}$.

Diethyl(2-chlorophenylamino)(3,4-dihydroxy-5-nitrophenyl)methylphosphonate (4n). Yield-84.6\%, colour-brown. ${ }^{1} \mathrm{H}-\mathrm{NMR}\left(300 \mathrm{MHz}, \mathrm{DMSO}-\mathrm{d}_{6}\right) \delta 10.2$ (br, 2H, -OH), 8.29$6.56(\mathrm{~m}, 6 \mathrm{H}, \mathrm{Ar}-\mathrm{H}), 5.45(\mathrm{~m}, 1 \mathrm{H}, \mathrm{N}-\mathrm{H}), 3.95$ (m, 1H, P-CH), $3.74\left(\mathrm{q}, 4 \mathrm{H}, \mathrm{P}-\mathrm{OCH}_{2}\right), 1.31\left(\mathrm{t}, 6 \mathrm{H}, \mathrm{P}-\mathrm{CCH}_{3}\right) .{ }^{31} \mathrm{P}-\mathrm{NMR}$ $\left(161.9 \mathrm{MHz}, \mathrm{DMSO}-\mathrm{d}_{6}\right) \delta 31.6 . \mathrm{M} / z: 430$ and 432 with $3: 1$ ratio. M.P. $175-178^{\circ} \mathrm{C}$.

Diethyl(2-chlorophenylamino)(4-hydroxy-3-methoxy-5-nitrophenyl)methylphosphonate (4o). Yield-90.2\%, colour-reddish brown, ${ }^{1} \mathrm{H}-\mathrm{NMR}\left(300 \mathrm{MHz}, \mathrm{DMSO}-\mathrm{d}_{6}\right) \delta 10.4(\mathrm{~s}, 1 \mathrm{H},-\mathrm{OH})$, 8.09-6.78 (m, 6H, Ar-H), $5.38(\mathrm{~m}, 1 \mathrm{H}, \mathrm{N}-\mathrm{H}), 4.12(\mathrm{~m}, 1 \mathrm{H}$, $\mathrm{P}-\mathrm{CH}), 3.76$ (q, 4H, P-OCH $\left.{ }_{2}\right), 2.91\left(\mathrm{~s}, 6 \mathrm{H},-\mathrm{OCH}_{3}\right), 1.17$ (t, 6H, P-CCH $\left.{ }_{3}\right) .{ }^{31} \mathrm{P}-\mathrm{NMR}\left(161.9 \mathrm{MHz}, \mathrm{DMSO}-\mathrm{d}_{6}\right) \delta 30.5$. $M / z: 444$ and 446 with $3: 1$ ratio. M.P. $173-175^{\circ} \mathrm{C}$.

2.5. Experimental Procedure for Antioxidant Activity. The antioxidant activity of the synthesized derivatives was evaluated using the DPPH free radical scavenging assay. $200 \mu \mathrm{L}$ of test sample solution $(100 \mu \mathrm{g} / \mathrm{mL})$ was added to $4 \mathrm{~mL}$ of $100 \mu \mathrm{M}$ methanolic DPPH. The mixture was incubated for 20 minutes at room temperature, and the absorbance at $517 \mathrm{~nm}$ was measured. BHT was used as standard. A blank was prepared without adding standard or test compound. Lowering the absorbance of the reaction mixture indicates higher free radical scavenging activity. The capability to scavenge the $\mathrm{DPPH}$ radical was calculated using the following equation:

$$
\text { DPPH scavenged }(\%)=\frac{\text { Abs control }- \text { Abs test }}{\text { Abs control }} \times 100 \text {, }
$$

where Abs control is the absorbance of the control reaction and Abs test is the absorbance in the presence of the test compounds. The antioxidant activities of the synthesized compounds are expressed comparing with standard BHT.

\section{Conclusion}

The synthesis of new $\alpha$-aminophosphonic acid esters was achieved in high yields through a one-pot three-component reaction process, a Kabachnik-Fields reaction. It involves the reactions among substituted anilines, substituted aromatic 
aldehydes, and dialkyl phosphites in dry toluene at reflux temperature, in the presence of Amberlite IRC-748 as catalyst. Their structures were established by elemental analysis IR, ${ }^{1} \mathrm{H}$ and ${ }^{31} \mathrm{P}-\mathrm{NMR}$, and mass spectral data. All the title compounds were screened for their antibacterial and antioxidant activity. Most of the compounds exhibited moderate antimicrobial activity, and for some the activity was fairly good.

\section{Acknowledgments}

The author is thankful to the Department of Chemistry, Central College Campus, Bangalore University for providing IR and elemental analysis, Indian Institute of Science, Bangalore, for providing NMR and mass spectra, and Sri. Venkateshwara Industries, Mandli Industrial Estate, Shimoga, for providing necessary facilities for the antibacterial and antioxidant activity tests.

\section{References}

[1] E. Breuer, The Chemistry of Organophosphorus Compounds, vol. 4, John Wiley \& Sons, New York, NY, USA, 1996.

[2] K. Srinivasulu, M. Anilkumar, C. Nagaraju, and C. S. Reddy, "Synthesis and bioactivity of some new 2-substituted-3,4-dihydro-1-(9H-carbazol-4-yloxy)methyl-3-[2-(2-methoxyphenoxy)ethyl]-1,3,2 $\lambda$ 5-oxazaphosphole 2-oxides, sulfides and selenides," ARKIVOC, vol. 2007, no. 14, pp. 100-109.

[3] T. K. Prakasha, R. O. Day, and R. R. Holmes, "New class of bicyclic oxyphosphoranes with an oxaphosphorinane ring: Molecular structures and activation energies for ligand exchange," Journal of the American Chemical Society, vol. 116, no. 18, pp. 8095-8104, 1994.

[4] C. Fest and K. J. Schmidt, The Chemistry of Organophosphorus Pesticides, vol. 12, Springer, 1982.

[5] M. S. Bhatia and P. Pawanjit, "Phosphorus containing heterocycles as fungicides: synthesis of 2,2' diphenylene chlorophosphonate and 2,2' diphenylene chlorothiophosphonate," Experientia, vol. 32, no. 9, p. 1111, 1976.

[6] P. N. Manne, S. D. Deshmukh, N. G. V. Rao, H. G. Dodale, and S. N. Tikar, "Efficacy of some insecticide against Helicoverpa armigera," Pestology, vol. 34, p. 65, 2000.

[7] D. Hendlin, E. O. Stapley, M. Jackson et al., "Phosphonomycin, a new antibiotic produced by strains of streptomyces," Science, vol. 166, no. 3901, pp. 122-123, 1969.

[8] A. M. Polozov and S. E. Cremer, "Synthesis of 2H-1,2-oxaphosphorin 2-oxides," Journal of Organometallic Chemistry, vol. 646, no. 1-2, pp. 153-160, 2002.

[9] V. P. Kukhar and H. R. Hudson, Aminophosphonic and Aminophosphinic Acids: Chemistry and Biological Activity, John Wiley \& Sons, New York, NY, USA, 2000.

[10] J. Huang and R. Chen, "HYPERLINK an overview of recent advances on the synthesis and biological activity of $\alpha$-aminophosphonic acid derivatives," Heteroatom Chemistry, vol. 11, pp. 480-492, 2000.

[11] L. D. Quin, A Guide to Organophosphorus Chemistry, vol. 11, Wiley, New York, NY, USA, 2000.

[12] J. Hiratake and J. ODA, "Aminophosphonic and aminoboronic acids as key elements of a transition state analogue inhibitor of enzymes," Bioscience, Biotechnology, and Biochemistry, vol. 61, no. 2, pp. 211-218, 1997.
[13] L. X. Xiao, K. Li, and D. Q. Shi, "A Convenient Synthesis and Herbicidal Activity of $N$-phosphonoalkylpyrazolo[4,3e] $[1,2,4]$-triazolo[1,5-d]pyrimidines," Phosphorus, Sulfur, and Silicon and the Related Elements, vol. 183, no. 12, pp. 3156-3165, 2008.

[14] M. C. Allen, W. Fuhrer, B. Tuck, R. Wade, and J. M. Wood, "Renin inhibitors. Synthesis of transition-state analogue inhibitors containing phosphorus acid derivatives at the scissile bond," Journal of Medicinal Chemistry, vol. 32, no. 7, pp. 16521661, 1989.

[15] F. R. Atherton, C. H. Hassall, and R. W. Lambert, "Synthesis and structure-activity relationships of antibacterial phosphonopeptides incorporating (1-aminoethyl)phosphonic acid and (aminomethyl)phosphonic acid," Journal of Medicinal Chemistry, vol. 29, no. 1, pp. 29-40, 1986.

[16] R. F. Pratt, "Inhibition of a class C $\beta$-lactamase by a specific phosphonate monoester," Science, vol. 246, no. 4932, pp. 917919, 1989.

[17] G. Lavielle, P. Hautefaye, C. Schaeffer, J. A. Boutin, C. A. Cudennec, and A. Pierré, "New $\alpha$-amino phosphonic acid derivatives of vinblastine: chemistry and antitumor activity," Journal of Medicinal Chemistry, vol. 34, no. 7, pp. 1998-2003, 1991.

[18] P. Kafarski and B. Lejczak, "Biological activity of aminophosphonic acids," Phosphorus, Sulfur, and Silicon and the Related Elements, vol. 63, no. 1-2, pp. 193-215, 1991.

[19] V. Rai and I. N. N. Namboothiri, "Enantioselective conjugate addition of dialkyl phosphites to nitroalkenes," Tetrahedron Asymmetry, vol. 19, no. 20, pp. 2335-2338, 2008.

[20] E. Kuliszewska, M. Hanbauer, and F. Hammerschmidt, "Preparation of $\alpha$-aminobenzylphosphonic acids with a stereogenic quaternary carbon atom via microscopically configurationally stable $\alpha$-aminobenzyllithiums," Chemistry A, vol. 14, no. 28, pp. 8603-8614, 2008.

[21] V. Coeffard, I. Beaudet, M. Evain, E. Le Grognec, and J. P. Quintard, "Preparation and transmetallation of enantioenriched $\alpha$ aminoorganostannanes derived from $N$-boc phenylglycinol: application to the synthesis of alafosfalin," European Journal of Organic Chemistry, vol. 2008, no. 19, pp. 3344-3351, 2008.

[22] R. A. Cherkasov and V. I. Galkin, "The Kabachnik-Fields reaction: synthetic potential and the problem of the mechanism," Russian Chemical Reviews, vol. 67, no. 10, pp. 857-882, 1998.

[23] N. S. Zefirov and E. D. Matveeva, "Catalytic Kabachnik-Fields reaction: new horizons for old reaction," ARKIVOC, vol. 1, no. 11, pp. 1-17, 2008.

[24] R. Jacquier, M. L. Hassani, C. Petrus, and F. Petrus, "Asymmetric synthesis of 1-aminoalkylphosphonic acids," Phosphorus, Sulfur, and Silicon and the Related Elements, vol. 81, no. 1-4, pp. 83-87, 1993.

[25] G. Jommi, G. Miglierini, R. Pagliarin, G. Sello, and M. Sisti, "Studies toward a model for predicting the diastereoselectivity in the electrophilic amination of chiral 1,3,2-oxazaphospholanes," Tetrahedron, vol. 48, no. 35, pp. 7275-7288, 1992.

[26] S. Hannesian and Y. Bennani, "Electrophilic amination and azidation of chiral $\alpha$-alkyl phosphonamides: asymmetric syntheses of $\alpha$-amino $\alpha$-alkyl phosphonic acids," Synthesis, vol. 1994, no. 12, pp. 1272-1276, 1994.

[27] S. E. Denmark, N. Chatani, and S. V. Pansare, "Asymmetric electrophilic amination of chiral phosphorus-stabilized anions," Tetrahedron, vol. 48, no. 11, pp. 2191-2208, 1992.

[28] A. N. Pudovik, "New method of synthesis of esters of phosphonocarboxylic acids and their derivatives," Doklady Akademii Nauk, vol. 85, pp. 349-351, 1952. 
[29] A. N. Pudovik and I. V. Konovalova, "Addition reactions of esters of phosphorus(III) acids with unsaturated systems," Synthesis, vol. 1979, no. 2, pp. 81-96, 1979.

[30] M. I. Kabachnik and T. Y. Medved, "New synthesis of aminophosphonic acids," Doklady Akademii Nauk, vol. 83, pp. 689692, 1952.

[31] E. K. Fields, "The synthesis of esters of substituted amino phosphonic acids," Journal of the American Chemical Society, vol. 74, no. 6, pp. 1528-1531, 1952.

[32] D. F. Wiemer, "Synthesis of nonracemic phosphonates," Tetrahedron, vol. 53, no. 49, pp. 16609-16644, 1997.

[33] B. C. Ranu and A. Hajra, "A simple and green procedure for the synthesis of $\alpha$-aminophosphonate by a one-pot threecomponent condensation of carbonyl compound, amine and diethyl phosphite without solvent and catalyst," Green Chemistry, vol. 4, no. 6, pp. 551-554, 2002.

[34] B. C. Ranu, A. Hajra, and U. Jana, "General procedure for the synthesis of $\alpha$-amino phosphonates from aldehydes and ketones using indium(III) chloride as a catalyst," Organic Letters, vol. 1, no. 8, pp. 1141-1143, 1999.

[35] M. R. Saidi and N. Azizi, "A new protocol for a one-pot synthesis of $\alpha$-amino phosphonates by reaction of imines prepared in situ with trialkylphosphites," Synlett, no. 8, pp. 1347-1349, 2002.

[36] N. Azizi and M. R. Saidi, "Lithium perchlorate-catalyzed threecomponent coupling: a facile and general method for the synthesis of $\alpha$-aminophosphonates under solvent-free conditions," European Journal of Organic Chemistry, vol. 2003, no. 23, pp. 4630-4633, 2003.

[37] S. Bhagat and A. K. Chakraborti, "An extremely efficient three-component reaction of aldehydes/ketones, amines, and phosphites (kabachnik-fields reaction) for the synthesis of $\alpha$ aminophosphonates catalyzed by magnesium perchlorate," The Journal of Organic Chemistry, vol. 72, no. 4, pp. 1263-1270, 2007.

[38] S. Bhagat and A. K. Chakraborti, "Zirconium(IV) compounds as efficient catalysts for synthesis of $\alpha$-aminophosphonates," The Journal of Organic Chemistry, vol. 73, no. 15, pp. 6029-6032, 2008.

[39] M. T. Maghsoodlou, S. M. Habibi Khorassani, R. Heydari, N. Hazeri, S. S. Sajadikhah, and M. Rostamizadeh, "Al(H2PO4)3 as an efficient and reusable catalyst for one-pot three-component synthesis of $\alpha$-amino phosphonates under solvent-free conditions," Chinese Journal of Chemistry, vol. 28, no. 2, pp. 285-288, 2010.

[40] Z. P. Zhan and J. P. Li, "Bismuth(III) chloride-catalyzed three, component coupling: synthesis of $\alpha$, amino phosphonates," Synthetic Communications, vol. 35, no. 19, pp. 2501-2504, 2005.

[41] Z. Rezaei, H. Firouzabadi, N. Iranpoor et al., "Design and one-pot synthesis of $\alpha$-aminophosphonates and bis $(\alpha$-aminophosphonates) by iron(III) chloride and cytotoxic activity," European Journal of Medicinal Chemistry, vol. 44, no. 11, pp. 4266-4275, 2009.

[42] F. Xu, Y. Q. Luo, J. T. Wu, Q. Shen, and H. Chen, "Facile one-pot synthesis of $\alpha$-amino phosphonates using lanthanide chloride as catalyst," Heteroatom Chemistry, vol. 17, no. 5, pp. 389-392, 2006.

[43] R. Ghosh, S. Maiti, A. Chakraborty, and D. K. Maiti, "In(OTf) catalysed simple one-pot synthesis of $\alpha$-amino phosphonates," Journal of Molecular Catalysis A, vol. 210, no. 1-2, pp. 53-57, 2004.

[44] S. Sobhani and Z. Tashrifi, "One-pot synthesis of primary 1-aminophosphonates: coupling reaction of carbonyl compounds, hexamethyldisilazane, and diethyl phosphite catalyzed by $\mathrm{Al}(\mathrm{OTf})_{3}$," Heteroatom Chemistry, vol. 20, no. 2, pp. 109-115, 2009.

[45] S. Sobhani and Z. Tashrifi, "Al(OTf)3 as an efficient catalyst for one-pot synthesis of primary diethyl 1-aminophosphonates under solvent-free conditions," Synthetic Communications, vol. 39, no. 1, pp. 120-131, 2009.

[46] M. Kasthuraiah, K. A. Kumar, C. S. Reddy, and C. D. Reddy, "Syntheses, spectral property, and antimicrobial activities of 6$\alpha$-amino dibenzo $[d, f][1,3,2]$ dioxaphosphepin 6-oxides," Heteroatom Chemistry, vol. 18, no. 1, pp. 2-8, 2007.

[47] S. Chandrasekhar, S. J. Prakash, V. Jagadeshwar, and C. Narsihmulu, "Three component coupling catalyzed by $\mathrm{TaCl}_{5}-\mathrm{SiO}_{2}$ : synthesis of $\alpha$-amino phosphonates," Tetrahedron Letters, vol. 42, no. 32, pp. 5561-5563, 2001.

[48] Y. P. Tian, F. Xu, Y. Wang, J. J. Tang, and H. L. Li, " $\mathrm{PPh}_{3}$-catalysed one-pot three-component syntheses of $\alpha$-aminophosphonates under solvent-free conditions," Journal of Chemical Research, vol. 2009, no. 2, pp. 78-80, 2009.

[49] A. K. Bhattacharya and K. C. Rana, "Amberlite-IR 120 catalyzed three-component synthesis of $\alpha$-amino phosphonates in onepot," Tetrahedron Letters, vol. 49, no. 16, pp. 2598-2601, 2008.

[50] J. Hou, J. Gao, and H. Zhang, " $\mathrm{NbCl}_{5}$ : an efficient catalyst for one-pot synthesis of $\alpha$-aminophosphonates under solvent-free conditions," Applied Organometallic Chemistry, vol. 25, no. 1, pp. 47-53, 2011.

[51] S. P. Bhimagouda, G. Krishnamurthy, H. S. Bhojyanaik, R. L. Prashant, and G. Manjunath, "Synthesis, characterization and antimicrobial studies of 2-(4-methoxy-phenyl)-5-methyl-4(2-arylsulfanyl-ethyl)-2,4-dihydro-[1,2,4] triazolo-3-ones and their corresponding sulfones," European Journal of Medicinal Chemistry, vol. 45, no. 8, pp. 3329-3334, 2010.

[52] R. J. Snow, A. Abeywardane, S. Campbell et al., "Hit-to-lead studies on benzimidazole inhibitors of ITK: Discovery of a novel class of kinase inhibitors," Bioorganic and Medicinal Chemistry Letters, vol. 17, no. 13, pp. 3660-3665, 2007.

[53] O. G. Ozden, E. Taner, G. Hakan, and Y. Sulhiye, "Synthesis and antimicrobial activity of some novel phenyl and benzimidazole substituted benzyl ethers," Bioorganic \& Medicinal Chemistry Letters, vol. 17, no. 8, pp. 2233-2236, 2007.

[54] S. P. Bhimagouda, G. Krishnamurthy, N. D. Shashikumar, M. R. Lokesh, and H. S. B. Naik, "Synthesis and antimicrobial activity of some [1,2,4]-triazole derivatives," Journal of Chemistry, vol. 2013, Article ID 462594, 7 pages, 2013.

[55] R. Diwedi, S. Alexandar, and M. J. N. Chandrasekar, "Rapid and efficient synthesis of microwave assisted some bis-1, 2, 4-triazole derivatives and their antioxidant and anti-inflammatory evaluation," Research Journal of Pharmaceutical, Biological and Chemical Sciences, vol. 2, no. 1, pp. 194-204, 2011. 

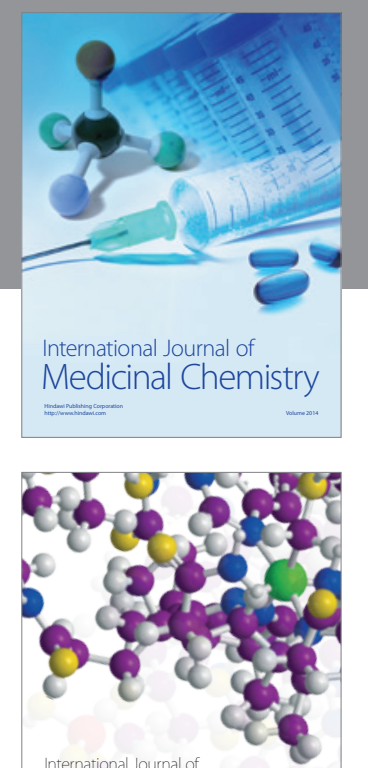

\section{Carbohydrate} Chemistry

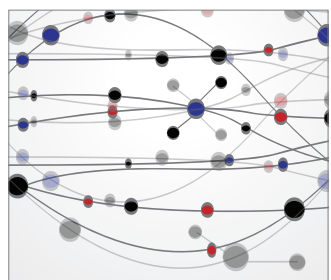

The Scientific World Journal
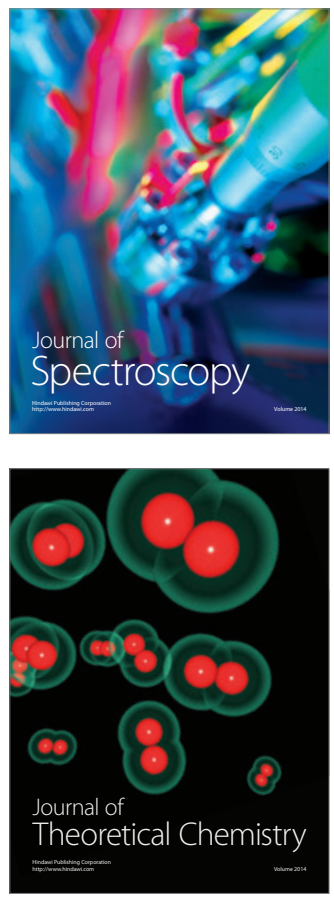
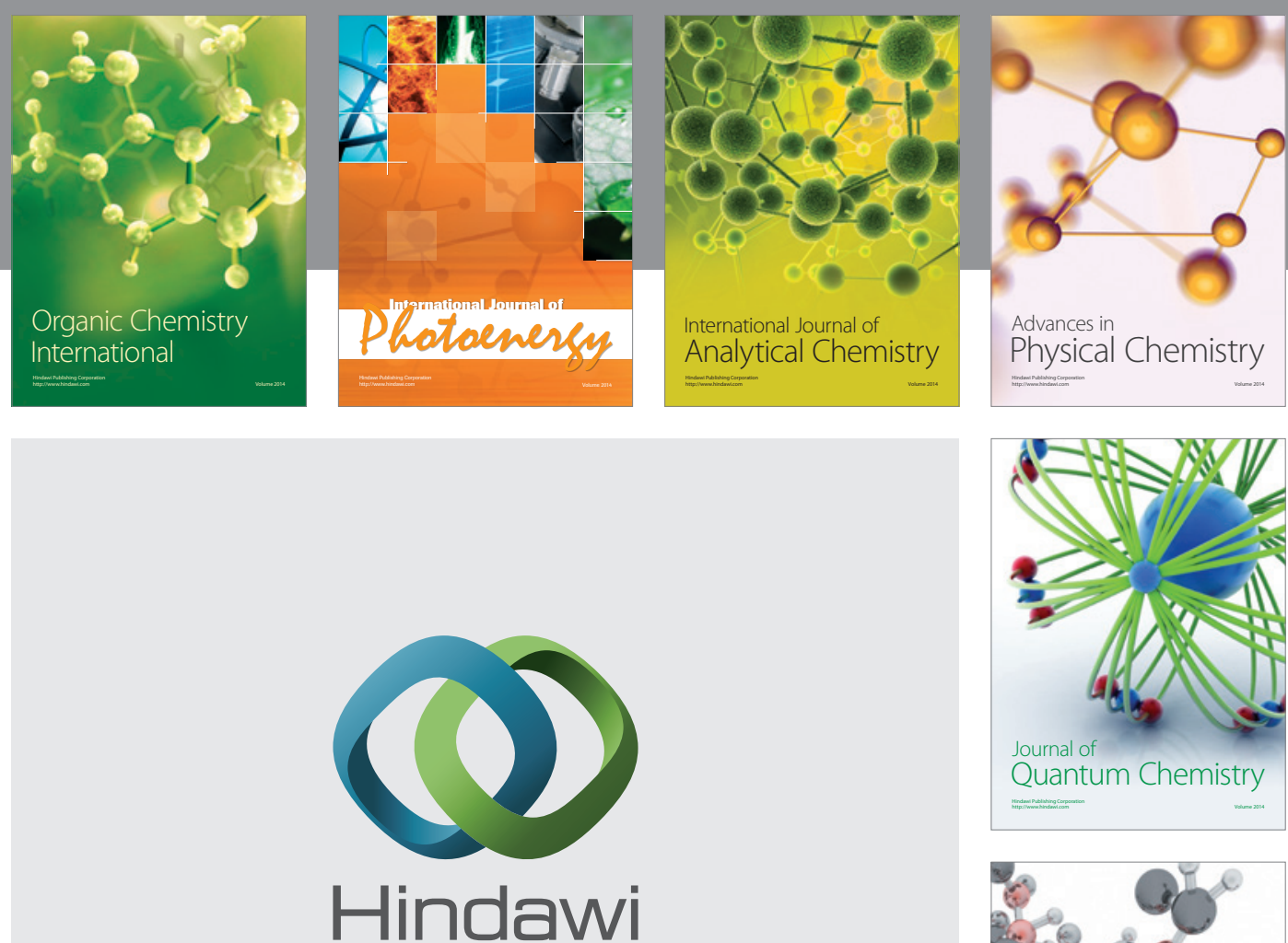

Submit your manuscripts at

http://www.hindawi.com

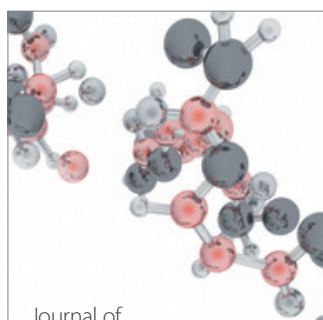

Analytical Methods

in Chemistry

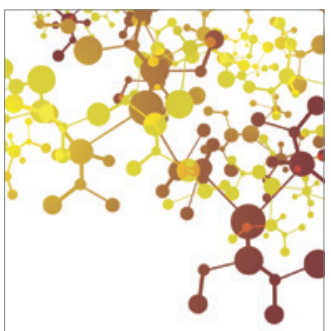

Journal of

Applied Chemistry

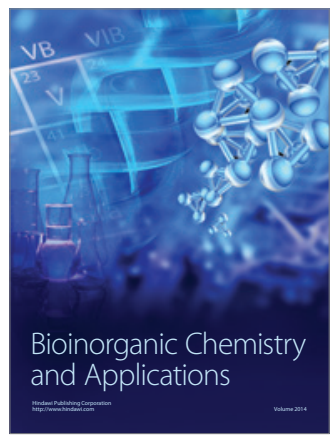

Inorganic Chemistry
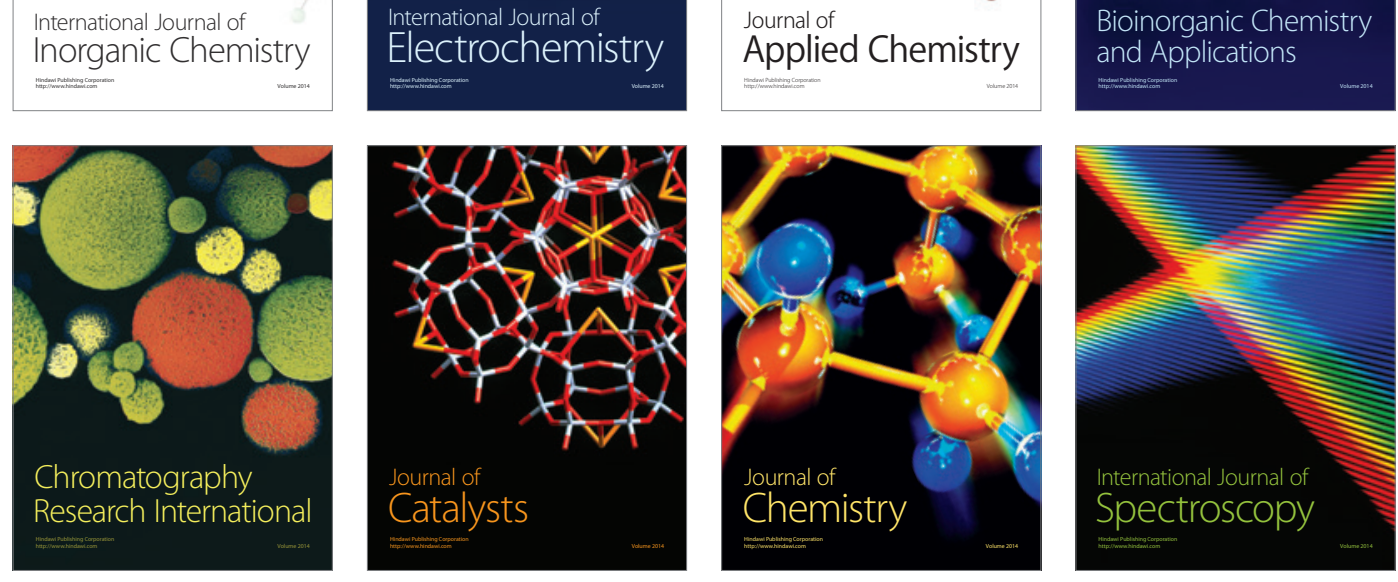